\title{
Coronal ejections from convective spherical shell dynamos
}

\author{
J. Warnecke ${ }^{1,2}$ P. J. Käpylä ${ }^{1,3}$ M. J. Mantere $^{3}$ and A. Brandenburg ${ }^{1,2}$ \\ ${ }^{1}$ Nordita, Roslagstullsbacken 23, SE-10691 Stockholm, Sweden, email: joern@nordita.org \\ ${ }^{2}$ Department of Astronomy, Stockholm University, SE-10691 Stockholm, Sweden \\ ${ }^{3}$ Department of Physics, PO BOX 64, FI-00014 Helsinki University, Finland
}

\begin{abstract}
We present a three-dimensional model of rotating convection combined with a simplified model of a corona in spherical coordinates. The motions in the convection zone generate a large-scale magnetic field which is sporadically ejected into the outer layers above. Our model corona is approximately isothermal, but it includes density stratification due to gravity.
\end{abstract}

Keywords. MHD, Sun: magnetic fields, Sun: coronal mass ejections (CMEs), turbulence

\section{Introduction}

The Sun sheds plasma into the heliosphere via coronal mass ejections (CMEs). There has been significant progress in the study of CMEs in recent years. In addition to improved observations from spacecrafts like SDO or STEREO, there have also been major advances in the field of numerical modeling of CME events. One of the main motivations for understanding the generation and dynamics of CMEs is to have more reliable predictions for space weather. CMEs can have strong impacts on Earth and can affect microelectronics aboard spacecrafts. However, an important side effect of CMEs is that through them the Sun sheds magnetic helicity from the convection zone which may prevent the solar dynamo from being quenched at high magnetic Reynolds numbers (Blackman \& Brandenburg, 2003).

In many CME models, footpoint motions of the magnetic field in the photosphere are taken from two-dimensional observations at the surface. This is an approximation to the full three-dimensional field generated by a turbulent dynamo. An alternative way of modeling CMEs would be to perform a 3-D convection simulation to generate the magnetic field and the photospheric motions self-consistently. However, convection zone and corona have very different timescales. In solar convection the dominant timescale varies from minutes to days. While this is short compared with the dynamo cycle, timescales in the solar corona can be even shorter because the Alfvén speed is large.

In earlier work (Warnecke \& Brandenburg 2010, Warnecke et al. 2011) we have established a two-layer model with a unified treatment of the convection zone and the solar corona in a single three-dimensional domain. In those models, magnetic fields were produced by turbulence from random helical forcing mimicking the effects of convection and rotation. This model was able to produce recurrent plasmoid ejections which are similar to observed eruptive features on the Sun. In Warnecke et al. (2012) we have developed this approach further and considered self-consistent convection instead, where differential rotation arises from the interaction of rotation and convection. Here we present some preliminary results of this study. We find the formation of a large-scale magnetic field, which is eventually ejected into the corona. This mechanism could play an important role for the formation of CMEs and flares. 


\section{The model}

As in Warnecke \& Brandenburg (2010) and Warnecke et al. (2011) a two-layer model is used. Our convection zone is similar to that in Käpylä et al. (2010, 2011). The domain is a segment of the Sun and is described in spherical polar coordinates $(r, \theta, \phi)$. We mimic the convection zone starting at radius $r=0.7 R$ and the solar corona until $r=1.5 R$, where $R$ denotes the solar radius, used from here on as our unit length. In the latitudinal direction, our domain extends from colatitude $\theta=15^{\circ}$ to $165^{\circ}$ and in the azimuthal direction from $\phi=0^{\circ}$ to $90^{\circ}$. We solve the equations of compressible magnetohydrodynamics,

$$
\begin{aligned}
\frac{\partial \boldsymbol{A}}{\partial t} & =\boldsymbol{U} \times \boldsymbol{B}+\eta \nabla^{2} \boldsymbol{A}, \\
\frac{\mathrm{D} \ln \rho}{\mathrm{D} t} & =-\boldsymbol{\nabla} \cdot \boldsymbol{U}, \\
\frac{\mathrm{D} \boldsymbol{U}}{\mathrm{D} t} & =\boldsymbol{g}-2 \boldsymbol{\Omega} \times \boldsymbol{U}+\frac{1}{\rho}(\boldsymbol{J} \times \boldsymbol{B}-\boldsymbol{\nabla} p+\boldsymbol{\nabla} \cdot 2 \nu \rho \mathbf{S}), \\
T \frac{\mathrm{D} s}{\mathrm{D} t} & =\frac{1}{\rho} \boldsymbol{\nabla} \cdot K \boldsymbol{\nabla} T+2 \nu \mathbf{S}^{2}+\frac{\mu_{0} \eta}{\rho} \boldsymbol{J}^{2}-\Gamma_{\text {cool }},
\end{aligned}
$$

where the magnetic field is given by $\boldsymbol{B}=\boldsymbol{\nabla} \times \boldsymbol{A}$ and thus obeys $\boldsymbol{\nabla} \cdot \boldsymbol{B}=0$ at all times. The vacuum permeability is given by $\mu_{0}$, whereas magnetic diffusivity and kinematic viscosity are given by $\eta$ and $\nu$, respectively. $\mathrm{S}_{i j}=\frac{1}{2}\left(U_{i ; j}+U_{j ; i}\right)-\frac{1}{3} \delta_{i j} \boldsymbol{\nabla} \cdot \boldsymbol{U}$ is the traceless rateof-strain tensor, and semicolons denote covariant differentiation, $\boldsymbol{\Omega}=\Omega_{0}(\cos \theta,-\sin \theta, 0)$ is the rotation vector, $K$ is the radiative heat conductivity and $\boldsymbol{g}=-G M \boldsymbol{r} / r^{3}$ is the gravitational acceleration. The fluid obeys the ideal gas law with $\gamma=5 / 3$ being the ratio of specific heats. We consider a setup in which the stratification is convectively unstable below $r=R$, whereas the region above is stably stratified and isothermal due to a cooling term $\Gamma_{\text {cool }}$ in the entropy equation. The $\Gamma_{\text {cool }}$ term is $r$ dependent and causes a smooth transition to the isothermal layer representing the corona.

The simulation domain is periodic in the azimuthal direction. For the velocity we use stress-free conditions at all other boundaries. For the magnetic field we adopt radial field conditions on the $r=1.5 R$ boundary and perfect conductor conditions on the $r=0.7 R$ and both latitudinal boundaries. Time is expressed in units of $\tau=\left(u_{\mathrm{rms}} k_{\mathrm{f}}\right)^{-1}$, which is the eddy turnover time in the convection zone. We employ the PENCIL Code $\dagger$, which uses sixth-order centered finite differences in space and a third-order Runge-Kutta scheme in time; see Mitra et al. (2009) for the extension to spherical coordinates.

\section{Results}

In this work we focus on a run which has fluid Reynolds number $\mathrm{Re}=3$, magnetic Reynolds number $\operatorname{Re}_{M}=32$ and Coriolis number $\mathrm{Co}=7$. We define the fluid and magnetic Reynolds number as $\mathrm{Re}=u_{\mathrm{rms}} / \nu k_{\mathrm{f}}$ and $\mathrm{Re}_{M}=u_{\mathrm{rms}} / \eta k_{\mathrm{f}}$, respectively, and the Coriolis number as Co $=2 \Omega_{0} / u_{\mathrm{rms}} k_{\mathrm{f}}$. After around 100 turnover times, the onset of large-scale dynamo action due to the convective motions is observed. The magnetic field reacts back on the fluid motions and causes saturation after around 200 turnover times. The saturation is combined with an oscillation of the magnetic field strength in the convection zone. The field reaches its maximum strength of about $60 \%$ of the equipartition field strength, $B_{\text {eq }}=\left(\mu_{0} \overline{\rho \boldsymbol{u}^{2}}\right)^{1 / 2}$, which is comparable with the values obtained in the forced turbulence counterparts both in Cartesian and spherical coordinates (Warnecke \& Brandenburg 2010, Warnecke et al. 2011). The magnetic field in rotating convection

$\dagger$ http://pencil-code.googlecode.com 

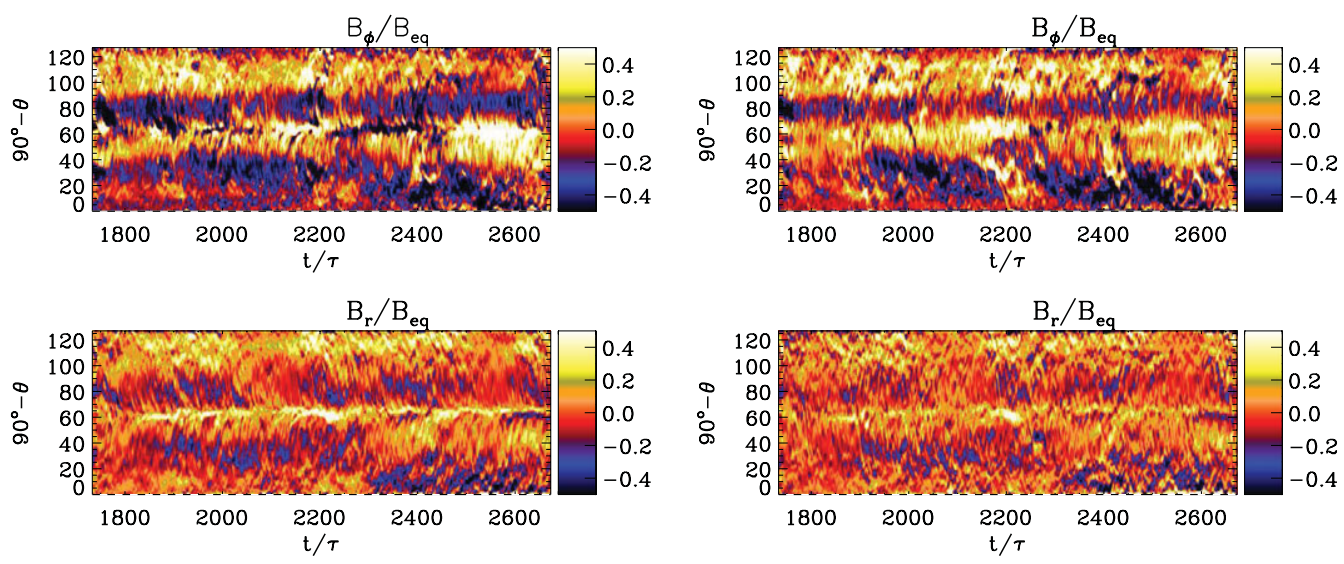

Figure 1. Variation of $\bar{B}_{\phi}$ and $\bar{B}_{r}$ in the convection zone at $r=0.89 R$ (left panel) and $r=0.79 R$ (right). Dark blue shades represent negative and light yellow positive values. The dotted horizontal lines show the location of the equator at $\theta=\pi / 2$. The magnetic field is normalized by the equipartition value.
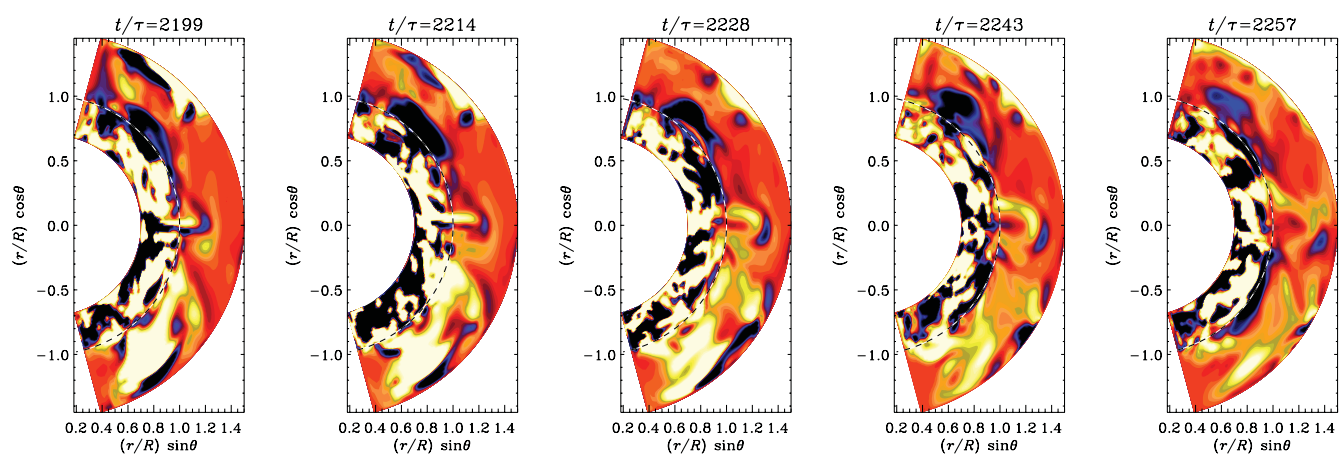

Figure 2. Time series of coronal ejections in spherical coordinates. The normalized current helicity, $\mu_{0} R \overline{\boldsymbol{J} \cdot \boldsymbol{B}} /\left\langle\overline{\boldsymbol{B}^{2}}\right\rangle_{t}$, is shown in a color-scale representation at different times; dark blue represents negative and light yellow positive values. The dashed horizontal lines show the location of the surface at $r=R$. Adapted from Warnecke et al. (2012).

seems to show certain migration properties. In Figure 1, we show the azimuthal $\left(\bar{B}_{\phi}\right)$ and radial $\left(\bar{B}_{r}\right)$ magnetic fields versus time $(t / \tau)$ and latitude $\left(90^{\circ}-\theta\right)$ for two different heights. The magnetic field emerges through the surface and is ejected as isolated structures. The dynamical evolution is clearly seen in the sequence of images of Figure 2, where the normalized current helicity $\left(\mu_{0} R \overline{\boldsymbol{J} \cdot \boldsymbol{B}} /\left\langle\overline{\boldsymbol{B}^{2}}\right\rangle_{t}\right)$ is shown. If one focuses on the region near the equator $(\theta=\pi / 2)$, a small yellow (i.e. positive) feature with a blue (negative) arch emerges through the surface to the outer atmosphere, where it leaves the domain through the outer boundary. This ejection does not occur as a single event-it rather shows recurrent behavior. We do not, however, find a clear periodicity in the ejection recurrence, like in earlier work. Even though the ejected structures are much smaller than in Warnecke et al. (2011), their shape is similar. However, the detection of an ejection with the aid of the current helicity is much more difficult in convection-driven simulations than in forced turbulence. In Figure 2, one can see large structures diffusing through the surface into the upper atmosphere at higher latitudes. These structures disturb the emergence of ejections and hamper their detection. These larger diffusive structures are also visible in Figure 3, where the normalized current density is averaged over two narrow 

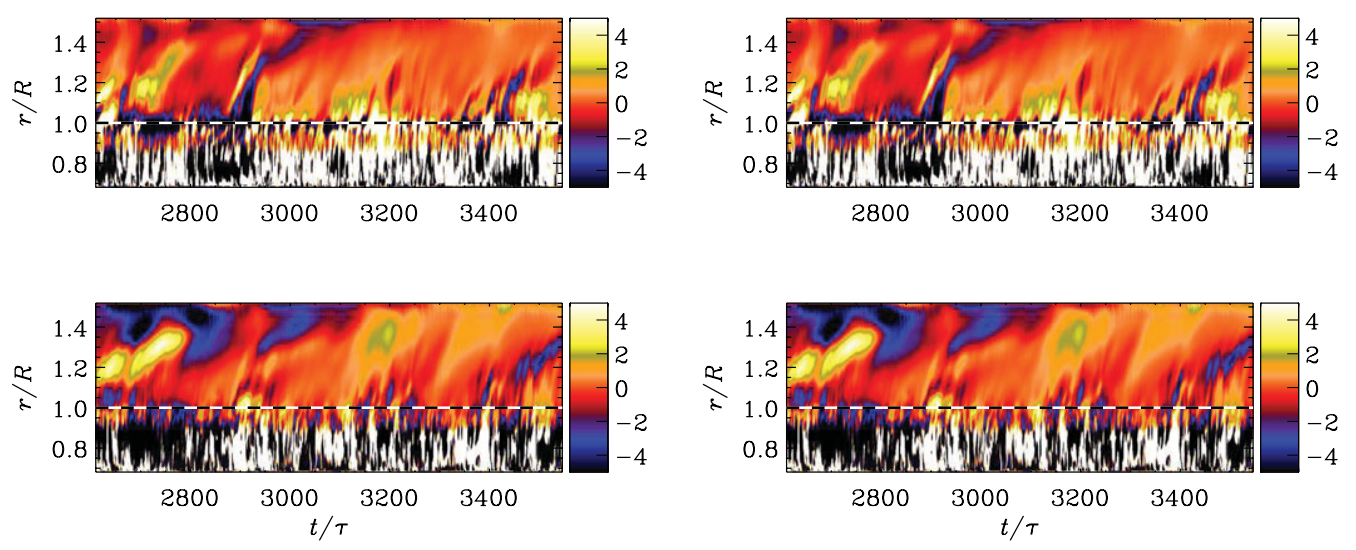

Figure 3. Dependence of the dimensionless ratio $\mu_{0} R \overline{\boldsymbol{J} \cdot \boldsymbol{B}} /\left\langle\overline{\boldsymbol{B}^{2}}\right\rangle_{t}$ on time $t / \tau$ and radius $r$ in terms of the solar radius. The top panels show a narrow band in $\theta$ in the northern hemisphere and the bottom ones in the southern hemisphere. We have also averaged in latitude from $4^{\circ}$ to $20^{\circ}$ (left panel) and $33^{\circ}$ to $46^{\circ}$ (right). Dark blue shades represent negative and light yellow positive values. The dotted horizontal lines show the location of the surface at $r=R$.

latitude bands on each hemisphere. The formation of these diffuse structures in the corona seem to get suppressed when the stratification of the system is increased. Nevertheless, ejections are still visible, for example around $t / \tau=1900, t / \tau=2200$ (see Figure 2) and $t / \tau=2400$.

In Warnecke et al. (2011) the sign of the current helicity in the northern (southern) hemisphere was negative (positive) in the interior and positive (negative) in the coronal part. However, in Figure 3 the current helicity shows different behaviors in the northern and southern hemispheres, and one cannot tell clearly the leading sign. This has to do with the much lower values of relative kinetic helicity $h_{\mathrm{rel}}(r, t)=\langle\boldsymbol{\omega} \cdot \boldsymbol{u}\rangle / \omega_{\mathrm{rms}} u_{\mathrm{rms}}$ in the convection simulations. Values of up to $h_{\text {rel }}=\mp 0.4$ are reached at some radii in the two hemispheres. In the forced turbulence simulations of our earlier work, we studied purely helical systems with nearly $h_{\text {rel }}=\mp 1$.

In summary, we have been able to advance our two-layer model approach by including self-consistent convection, which generates the magnetic field and eventually drives ejections. Unlike our earlier work, the ejections occur now non-periodically and through smaller structures, which is now closer to the behavior displayed by the Sun. Furthermore, detailed investigations covering a wider range of magnetic and kinetic Reynolds number as well as rotation rates show promising results; see Warnecke et al. (2012) for details.

\section{References}

Blackman, E. G. \& Brandenburg, A. 2003, ApJ, 584, L99

Käpylä, P. J., Korpi, M. J., Brandenburg, A., Mitra, D., \& Tavakol, R. 2010, AN, 331, 73

Käpylä, P. J., Korpi, M. J., Guerrero, G., Brandenburg, A., \& Chatterjee, P. 2011, A\&A, 531, A162

Mitra, D., Tavakol, R., Brandenburg, A., \& Moss, D. 2009, ApJ, 697, 923

Warnecke, J. \& Brandenburg, A. 2010, A\&A, 523, A19

Warnecke, J., Brandenburg, A., \& Mitra, D. 2011, A\&A, 534, A11

Warnecke, J., Käpylä, P. J., Mantere, M. J., \& Brandenburg, A. 2012, Sol. Phys. (in press, arxiv:1112.0505) 


\section{Discussion}

DANiEl Gómez: Why do you use the current helicity and not the magnetic helicity?

JÖRN WARNECKE: The spectra of current helicity and magnetic helicity are related to each other by a $k^{2}$ factor, where $k$ is the wavenumber. The current helicity integrated over all wavenumbers is equal to the integrated magnetic helicity spectrum weighed toward large $k$. Current helicity can therefore be regarded as a proxy of magnetic helicity at small scales. Furthermore, unlike magnetic helicity, current helicity is gauge-invariant and therefore a physically meaningful quantity.

JANET Luhmann: There are different flavors of CMEs. Which ones do you model here: streamers, blow outs? Comment: There is very clear cycling of the helicity of the activity clouds. It would be interesting to follow these results to complement your model.

JÖRN WARNECKE: We do not model specific CMEs. Rather, the objective of this project is to link dynamo-generated magnetic fields to driving ejecta from its surface. Our model is not sufficiently realistic to make meaningful comparisons with detailed observations. Nevertheless, the visual similarity with actual CMEs inspires us to look more carefully at certain features that might relate our ejecta with CMEs.

Francesco ZuCCARELLO: Why are some CMEs deflected?

JÖRN WARNECKE: Even though the boundary condition for the magnetic field is an open one (vertical field condition), the condition for the velocity is a closed one (stress free). So the mass associated with CMEs cannot get out and must fall back. We are currently working on open boundary conditions allowing mass to escape. This would also allow a solar wind to develop.

JON LINKER: For CMEs on the Sun associated with active regions: you do sometimes see a CME that occurs right after an active region emerges, but very often the active region hangs around and decays before it produces a CME. It sort of appears in your model that you see the eruption of the CME through the photosphere and the whole thing is blown away completely. I am wondering if there needs to be more restraining (coronal) field there to begin with, otherwise many of these so called CMEs might just be emergence of an active region.

JÖRN WARNECKE: It is right, that we do not include constraining coronal fields in our models. In addition our density stratification and the radiation effects of the photosphere and chromosphere are very simplified. Nevertheless, it is interesting to interpret our results as the emergence of an active region, and we shall look more closely into this. 